\title{
Editorial
}

\section{Wearable Wireless Devices}

\author{
Qammer Hussain Abbasi ${ }^{1,2, *\left(\mathbb{D}, \text { Hadi Heidari }^{1}{ }^{1} \text { and Akram Alomainy }\right.}{ }^{2}$ \\ 1 James Watt School of Engineering, University of Glasgow, Glasgow G12 8QQ, UK \\ 2 School of Electronic Engineering and Computer Science, Queen Mary University of London, \\ London E1 4NS, UK \\ * Correspondence: qammer.abbasi@glasgow.ac.uk
}

Received: 5 June 2019; Accepted: 24 June 2019; Published: 29 June 2019

\section{Introduction}

With the growing interest in the use of technology in daily life, the potential of using wearable wireless devices across multiple segments, e.g., healthcare, sports, child monitoring, military, emergency, consumer electronics, etc., is rapidly increasing. It is predicted that there will be multibillion wearable sensors by 2025 , with over $30 \%$ of them being new types of sensors that are just beginning to emerge. This Special Issue will be focused on wireless wearable and implantable system, flexible textile-based electronics, bio-electromagnetics, antennas and propagation, RF circuits, sensor, security of wearables and implantable system, nano-bio communication and electromagnetic sensing.

\section{Contributions}

The special issue consists of 10 contributions in the area of wearable wireless devices:

In the paper, 'Reservoir Computing Based Echo State Networks for Ventricular Heart Beat Classification' [1] a Reservoir Computing (RC) based Echo State Networks (ESNs) for ventricular heartbeat classification based on a single Electrocardiogram (ECG) lead has been proposed. The proposed method was especially designed for Medical Internet of Things (MIoT) devices, for instance wearable wireless devices for ECG monitoring or ventricular heart beat detection systems and so on. The performance of the proposed model was evaluated using the MIT-BIH-AR dataset and it achieved excellent results.

In the paper, 'A Real Time and Lossless Encoding Scheme for Patch Electrocardiogram Monitors' [2] an ECG encoding scheme for joint lossless data compression and heartbeat detection to minimize the circuit footprint size and power consumption of a patch electrocardiogram (ECG) monitors (PEMs) has been proposed. The proposed encoding scheme supports two operation modes: fixed-block mode and dynamic-block mode. Dynamic-block mode provides heartbeat detection accuracy at a rate higher than $98 \%$. Fixed-block mode was also implemented on the field-programmable gate array, and could be used as a chip for using analog-to-digital convertor-ready signals as an operation clock.

In the paper, 'Foot-Mounted Inertial Measurement Units-Based Device for Ankle Rehabilitation' [3], authors presented an inertial measurement units (IMU)-based physical interface for measuring the foot attitude, and a graphical user interface that acts as a visual guide for patient rehabilitation. According to the results, more consistent rehabilitation could be achieved by providing feedback on foot angular position during therapy procedures.

In the paper, 'Chronic Obstructive Pulmonary Disease Warning in the Approximate Ward Environment' [4] the usage of modern 5G C-Band sensing for health care monitoring has been proposed. The focus of this research was to monitor the respiratory symptoms for COPD (Chronic Obstructive Pulmonary Disease). The 5G sensing technique enhances the sensing performance for the health care sector by monitoring the amplitude information for different respiratory activities of a patient using the above-mentioned devices. 
In the paper, 'Movement Noise Cancellation in Second Derivative of Photoplethysmography Signals with Wavelet Transform and Diversity Combining' [5] authors proposed an algorithm to remove movement noise from second derivative of photoplethysmography (SDPPG) signals. Experiment results show that the proposed algorithm outperforms the previous filter-based algorithm, and that movement noise with $30 \%$ time duration can be reduced by up to $70.89 \%$.

In the paper, 'An Anonymous Mutual Authenticated Key Agreement Scheme for Wearable Sensors in Wireless Body Area Networks', [6] authors proposed that Li et al. lightweight protocol for wearable sensors in wireless body area networks is still vulnerable to three types of attacks i.e., the offline identity guessing attack, the sensor node impersonation attack and the hub node spoofing attack. Authors present a secure scheme that addresses these problems, and retains similar efficiency in wireless sensors nodes and mobile phones.

In the paper, 'Respiration Symptoms Monitoring in Body Area Networks' [7] authors presented a framework that monitors particular symptoms such as respiratory conditions (abnormal breathing pattern) experienced by hyperthyreosis, sleep apnea, and sudden infant death syndrome (SIDS) patients. The rhythmic patterns extracted using S-Band sensing present the periodic and non-periodic waveforms that correspond to normal and abnormal respiratory conditions, respectively.

In the paper, 'Internet of Things for Sensing: A Case Study in the Healthcare System' [8], authors did a pilot study, to look at narcolepsy, a disorder in which individuals lose the ability to regulate their sleep-wake cycle. Using S-band sensing Classification and validation of various human activities such as walking, sitting on a chair, push-ups, and narcolepsy sleep episodes are done using support vector machine, K-nearest neighbor, and random forest algorithms. The measurement and evaluation were carried out several times with classification values of accuracy, precision, recall, specificity, Kappa, and F-measure of more than $90 \%$ that were achieved when delineating sleep attacks.

In the paper, 'Emergency-Prioritized Asymmetric Protocol for Improving QoS of Energy-Constraint Wearable Device in Wireless Body Area Networks' [9], authors proposed a new MAC protocol to satisfy the higher energy efficiency of nodes than coordinator by designing the asymmetrically energy-balanced model between nodes and coordinator. The proposed scheme loads the unavoidable energy consumption into the coordinator instead of the nodes to extend their lifetime. Additionally, the scheme also provides prioritization for the emergency data transmission with differentiated Quality of Service (QoS). For the evaluations, IEEE 802.15.6 was used for comparison.

In the paper, 'Virtual Reality-Wireless Local Area Network: Wireless Connection-Oriented Virtual Reality Architecture for Next-Generation Virtual Reality Devices' [10], authors carefully examine the feasibility of wireless VR over WLANs, and proposed an efficient wireless multiuser VR communication architecture, as well as a communication scheme for VR. Extensive simulations have been performed to corroborate the outstanding performance of the proposed scheme.

The editors hope that this special issue will benefit the scientific community and contribute to the knowledge base.

Acknowledgments: The editors would take this opportunity to applaud the contribution of the authors to this special issue. Efforts of the reviewers to enhance the quality of the manuscripts is also much appreciated.

Conflicts of Interest: The authors declare no conflict of interest.

\section{References}

1. Mastoi, Q.; Wah, T.; Gopal Raj, R. Reservoir Computing Based Echo State Networks for Ventricular Heart Beat Classification. Appl. Sci. 2019, 9, 702. [CrossRef]

2. Fang, H.; Lu, C. A Real Time and Lossless Encoding Scheme for Patch Electrocardiogram Monitors. Appl. Sci. 2018, 8, 2379. [CrossRef]

3. Gómez-Espinosa, A.; Espinosa-Castillo, N.; Valdés-Aguirre, B. Foot-Mounted Inertial Measurement Units-Based Device for Ankle Rehabilitation. Appl. Sci. 2018, 8, 2032. [CrossRef]

4. Zhang, Q.; Haider, D.; Wang, W.; Shah, S.; Yang, X.; Abbasi, Q. Chronic Obstructive Pulmonary Disease Warning in the Approximate Ward Environment. Appl. Sci. 2018, 8, 1915. [CrossRef] 
5. Ban, D.; Shahid, S.; Kwon, S. Movement Noise Cancellation in Second Derivative of Photoplethysmography Signals with Wavelet Transform and Diversity Combining. Appl. Sci. 2018, 8, 1531. [CrossRef]

6. Chen, C.; Xiang, B.; Wu, T.; Wang, K. An Anonymous Mutual Authenticated Key Agreement Scheme for Wearable Sensors in Wireless Body Area Networks. Appl. Sci. 2018, 8, 1074. [CrossRef]

7. Liu, L.; Shah, S.; Zhao, G.; Yang, X. Respiration Symptoms Monitoring in Body Area Networks. Appl. Sci. 2018, 8, 568. [CrossRef]

8. Shah, S.; Ren, A.; Fan, D.; Zhang, Z.; Zhao, N.; Yang, X.; Luo, M.; Wang, W.; Hu, F.; Rehman, M.; et al. Internet of Things for Sensing: A Case Study in the Healthcare System. Appl. Sci. 2018, 8, 508. [CrossRef]

9. Lee, J.; Kim, S. Emergency-Prioritized Asymmetric Protocol for Improving QoS of Energy-Constraint Wearable Device in Wireless Body Area Networks. Appl. Sci. 2018, 8, 92. [CrossRef]

10. Ahn, J.; Kim, Y.; Kim, R. Virtual Reality-Wireless Local Area Network: Wireless Connection-Oriented Virtual Reality Architecture for Next-Generation Virtual Reality Devices. Appl. Sci. 2018, 8, 43. [CrossRef]

(C) 2019 by the authors. Licensee MDPI, Basel, Switzerland. This article is an open access article distributed under the terms and conditions of the Creative Commons Attribution (CC BY) license (http://creativecommons.org/licenses/by/4.0/). 